\title{
Two-dimensional individual clustering model
}

\author{
Elissar Nasreddine \\ Institut de Mathématiques de Toulouse, Université de Toulouse, \\ F-31062 Toulouse cedex 9, France \\ e-mail: elissar.nasreddine@math.univ-toulouse.fr \\ January 5, 2019
}

\begin{abstract}
This paper is devoted to study a model of individual clustering with two specific reproduction rates in two space dimensions. Given $q>2$ and an initial condition in $W^{1, q}(\Omega)$, the local existence and uniqueness of solution have been shown in [6]. In this paper we give a detailed proof of existence of global solution.
\end{abstract}

\section{Introduction}

In the present work, we deal with a model of individual dispersing of individual with an additional aggregation mechanism introduced in [5]. Given a sufficiently smooth function $E$, parameters $\delta \in(0,1), \varepsilon \geq 0$ and $r \geq 0$, the equations take the form

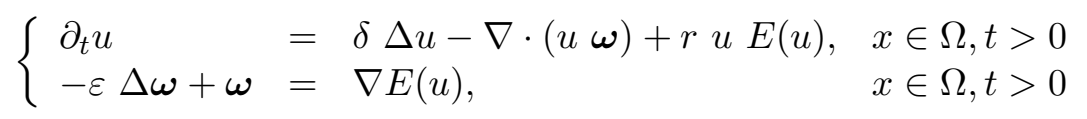

in an open bounded domain $\Omega \subset \mathbb{R}^{2}$, where $u(t, x)>0, \boldsymbol{\omega}(t, x) \in \mathbb{R}^{2}$ and $E$ denote the population density, the average velocity of dispersing individuals, and the individual net reproduction rate, respectively. In this model, the individuals are assumed to disperse randomly in space $(\delta \Delta u)$ with a bias $-\nabla \cdot(u \boldsymbol{\omega})$ in the direction of increasing reproduction rate, the term $\varepsilon \Delta \boldsymbol{\omega}$ acting as a mollifier to smooth out any sharp local variation in $\nabla E(u)$.

In [5], we supplement (1) with no-flux boundary conditions

$$
\partial_{\boldsymbol{n}} u=\boldsymbol{n} \cdot \boldsymbol{\omega}=0, \quad x \in \partial \Omega, t \geq 0,
$$

where $\boldsymbol{n}$ is the outward unit normal of $\partial \Omega$ and $\partial_{\boldsymbol{n}} u=\boldsymbol{n} \cdot \nabla u$. However, to guarantee the well-posedness of the elliptic system for $\boldsymbol{\omega}$ in two space dimensions, we should append the following condition given in $[\underline{3}, \underline{4}, \underline{7}]$

$$
\partial_{\boldsymbol{n}} \boldsymbol{\omega} \times \boldsymbol{n}=0, \quad x \in \partial \Omega, t \geq 0,
$$

where $\partial_{\boldsymbol{n}} \boldsymbol{\omega}=\left(\partial_{\boldsymbol{n}} \omega_{1}, \partial_{\boldsymbol{n}} \omega_{2}\right)=\left(\boldsymbol{n} \cdot \nabla \omega_{1}, \boldsymbol{n} \cdot \nabla \omega_{2}\right)$ for the vector field $\boldsymbol{\omega}=\left(\omega_{1}, \omega_{2}\right)$. in other words, (3) means that $\partial_{\boldsymbol{n}} \boldsymbol{\omega}$ is parallel to $\boldsymbol{n}$, where $\boldsymbol{v} \times \boldsymbol{u}=v_{1} u_{2}-u_{1} v_{2}$.

Given $q>2$, and an initial condition $u_{0} \in W^{1, q}(\Omega)$, the existence and uniqueness of a nonnegative and maximal solution of (1), (2) and (ㄱ) have been shown in [6], and the purpose of this paper is to prove the global existence of solution when $E(u)$ has the two specific forms suggested in [5], namely

$$
E(u)=(1-u)(u-a)
$$


for some $a \in(0,1)$, or

$$
E(u)=1-u \text {. }
$$

For these choices of reproduction rates, global existence has been shown in [6] in one space dimension and the purpose of this work is to prove that the solutions are global as well in two space dimensions. As in the one-dimensional case, the starting point of the analysis is an $L^{\infty}\left(L^{2}\right)$ estimate on $u$ and an $L^{2}$ estimate on $\nabla \cdot \boldsymbol{\omega}$. Combining the latter with Gagliardo-Nirenberg inequality gives $L^{\infty}\left(L^{p}\right)$ estimates on $u$ for any $p>2$. This then allow us to obtain an $L^{\infty}\left(L^{2}\right)$ bound on $\nabla u$ which in turn gives an $L^{\infty}$ bound on $\boldsymbol{\omega}$ by elliptic regularity.

The paper is organized as follows. In section 2, we state the global existence results, and focus on the two specific forms of $E$ : The "bistable case" (4) see Theorem 2.2, and the "monostable case" ( 5 ), see Theorem 2.3. In section 3, we recall the local existence result obtained in [6] and we give some properties of the elliptic system for $\boldsymbol{\omega}$. In section 4 , we turn to the global existence issue in the bistable case. The proof starts from the $L^{\infty}\left(L^{2}\right)$ estimate for $u$, an $L^{2}$ estimate for $\boldsymbol{\omega}$ and an $L^{2}$ estimate on $\nabla \cdot \boldsymbol{\omega}$ obtained from a suitable cancellation between the coupling terms in the $u$ and $\boldsymbol{\omega}$ equations, then, for $p>2$, we derive an $L^{\infty}\left(L^{p}\right)$ estimate for $u$. Then we use Lemma A.1 of [8] to derive an $L^{\infty}$ estimate of $u$ and we end the proof by an $L^{\infty}\left(L^{q}\right)$ estimate on $\nabla u$. This ensures global existence. In section 5 , we prove the global existence in the monostable case. The proof is quite similar to that of the previous case, except for the first estimate.

\section{Main result}

We first define the notion of solution to (1)-( $\underline{3})$ to be used in this paper.

Definition 2.1. Let $T>0, q>2$, and an initial condition $u_{0} \in W^{1, q}(\Omega)$. A strong solution of (1)-(3) on $[0, T)$ is a function

$$
u \in C\left([0, T), W^{1, q}(\Omega)\right) \cap C\left((0, T), W^{2, q}(\Omega)\right),
$$

such that

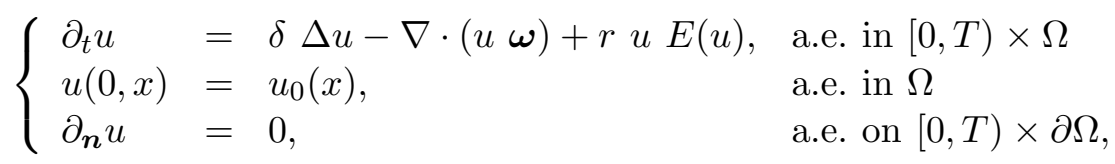

where, for all $t \in[0, T), \boldsymbol{\omega}(t)$ is the unique solution in $W^{2, q}(\Omega)$ of

$$
\left\{\begin{array}{lll}
-\varepsilon \Delta \boldsymbol{\omega}(t)+\boldsymbol{\omega}(t) & =\nabla E(u(t)) & \text { a.e. in } \Omega \\
\boldsymbol{\omega}(t) \cdot \boldsymbol{n}=\partial_{\boldsymbol{n}} \boldsymbol{\omega}(t) \times \boldsymbol{n} & =0 & \text { a.e. on } \partial \Omega
\end{array}\right.
$$

In the following theorem we give the global existence of solution to (1)-( 3$)$ in the bistable case, that is when $E(u)=(1-u)(u-a)$, for some $a \in(0,1)$.

Theorem 2.2. Let $q>2$, and assume that $u_{0}$ is a nonnegative function in $W^{1, q}(\Omega)$, and $E(u)=(1-u)(u-a)$ for some $a \in(0,1)$. Then (1)-(3) has a global nonnegative solution $u$ in the sense of Definition 2.1 .

The proof starts with a suitable cancellation of the coupling terms in the two equations which gives an estimate for $u$ in $L^{\infty}\left(L^{2}\right)$ and for $\nabla \cdot \boldsymbol{\omega}$ in $L^{2}$. Using the Gagliardo-Nirenberg 
inequality (13) we derive, for $p>2$ an $L^{\infty}\left(L^{p}\right)$ estimate for $u$. Then by the regularity properties of the second equation in (1) we obtain an $L^{\infty}$ bound of $\boldsymbol{\omega}$. Combining these estimates and Lemma A.1 of [8] provide us with an $L^{\infty}$ estimate for $u$ which is used to show an $L^{\infty}\left(L^{q}\right)$ estimate for $\nabla u$. This proves that the solution cannot explode in finite time.

Next, we turn to the global existence issue in the monostable case, that is when $E(u)=$ $1-u$.

Theorem 2.3. Let $q>2$, and assume that $u_{0}$ is a nonnegative function in $W^{1, q}(\Omega)$, and $E(u)=(1-u)$. Then $(1)-(3)$ has a global nonnegative solution $u$ in the sense of Definition 2.1.

The proof of the previous theorem follows the same lines as that of Theorem 2.2. As in the bistable case, there is a cancellation between the two equations which provide us an $L^{\infty}(L \log L)$ bound on $u$ and an $L^{2}$ bound for $\nabla \cdot \boldsymbol{\omega}$ as a starting point.

\section{Well-posedness}

Throughout this paper and unless otherwise stated, we assume that

$$
\delta \in(0,1), \varepsilon>0, r \geq 0 .
$$

We first recall some properties of the strong solution of the following system,

$$
\left\{\begin{array}{lll}
-\varepsilon \Delta \boldsymbol{\omega}+\boldsymbol{\omega} & =f, & \text { in } \Omega, \\
\boldsymbol{\omega} \cdot \boldsymbol{n} & =0, & \text { on } \partial \Omega, \\
\partial_{\boldsymbol{n}} \boldsymbol{\omega} \times \boldsymbol{n} & =0, & \text { on } \partial \Omega,
\end{array}\right.
$$

where $f \in\left(L^{p}(\Omega)\right)^{2}$ and $p>1$. The strong solutions of ( $\left.\underline{8}\right)$ is solving ( $\left.\underline{8}\right)$ a.e. in $\Omega$. In this direction the existence and uniqueness of the strong solution to $(\underline{8})$ are proved in [7]:

Theorem 3.1. For $f \in\left(L^{p}(\Omega)\right)^{2}$ with $1<p<\infty$, () has a unique solution in $\left(W^{2, p}(\Omega)\right)^{2}$ such that

$$
\|\boldsymbol{\omega}\|_{W^{2, p}} \leq \frac{K(p)}{\varepsilon}\|f\|_{p}
$$

where $K(p)=K(p, \Omega)$.

In other words, the strong solution has the same regularity as elliptic equations with classical boundary conditions.

Thanks to [6] we recall the existence and uniqueness result of the maximal solution of $(\underline{1})-(\underline{3})$.

Theorem 3.2. We assume that $E \in C^{2}(\mathbb{R})$, let $p>2$ and a nonnegative function $u_{0} \in W^{1, p}(\Omega)$. Then, for some $T_{\max } \in(0, \infty]$, there is a unique nonnegative maximal solution

$$
u \in C\left(\left[0, T_{\max }\right), W^{1, p}(\Omega)\right) \cap C\left(\left(0, T_{\max }\right), W^{2, p}(\Omega)\right)
$$

to (1)-(3) in the sense of Definition 2.1. Moreover, if for each $T>0$, there is $C(T)$ such that

$$
\|u(t)\|_{W^{1, p}} \leq C(T), \text { for all } t \in[0, T] \cap\left[0, T_{\max }\right),
$$


then $T_{\max }=\infty$. In addition, $u$ satisfies

$$
u(t, x)=\left(e^{t(\delta \Delta)} u_{0}(x)\right)+\int_{0}^{t} e^{(t-s)(\delta \Delta)}[-\nabla \cdot(u \boldsymbol{\omega})+r u E(u)](s, x) d s,
$$

for $(t, x) \in\left[0, T_{\max }\right] \times \Omega$, where $\left(e^{t(\delta \Delta)}\right)$ denotes the semigroup generated in $L^{p}(\Omega)$ by $\delta \Delta$ with homogeneous Neumann boundary conditions.

We recall that there is $C>0$ such that

$$
\left\|e^{t(\delta \Delta)} v\right\|_{W^{1, p}} \leq C\|v\|_{W^{1, p}} \text {, and }\left\|\nabla e^{t(\delta \Delta)} v\right\|_{p} \leq C \delta^{-\frac{1}{2}} t^{-\frac{1}{2}}\|v\|_{p} .
$$

Also in several places we shall need the following Gagliardo-Nirenberg inequality

$$
\|u\|_{p} \leq C\|u\|_{W^{1,2}}^{\theta}\|u\|_{q}^{1-\theta}, \quad \text { with } \theta=\frac{p-q}{p}, u \in W^{1,2}(\Omega)
$$

which holds for all $p \geq 1$ and $q \in[1, p]$. Also we use the following singular Gronwall lemma (see [1, Theorem 3.3.1]).

Lemma 3.3. Given $\alpha, \beta \in[0,1)$, there exists a positive constant $c:=c(\alpha, \beta)$ such that the following is true:

If $f:(0, T) \longrightarrow \mathbb{R}$ satisfies

$$
\left[t \mapsto t^{\beta} f(t)\right] \in L_{\mathrm{loc}}^{\infty}((0, T), \mathbb{R}),
$$

and

$$
f(t) \leq A t^{-\beta}+B \int_{0}^{t} \frac{1}{(t-s)^{\alpha}} f(s) d s, \text { a.a.t } \in(0, T),
$$

where $A$ and $B$ are positive constants, then $f(t) \leq C(T)$, for all $t \in(0, T)$, where $C$ depends only on $T, \alpha, \beta$, and $\gamma$.

\section{Global existence}

\subsection{The bistable case: $E(u)=(1-u)(u-a)$}

We recall the system

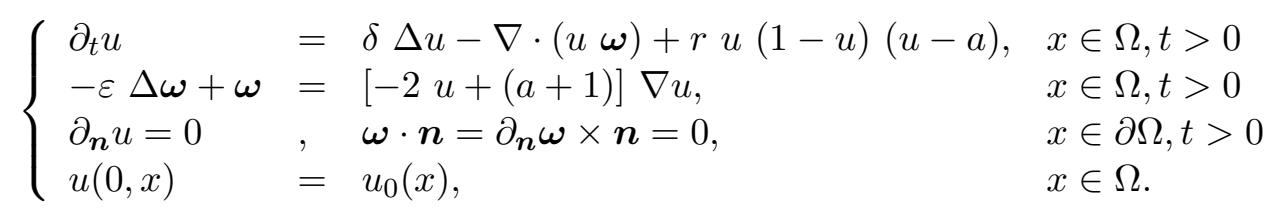

for a some $a \in(0,1)$, and $u_{0} \in W^{1, q}(\Omega)$ for some $q>2$.

Since $E \in C^{2}(\mathbb{R})$, Theorem 3.2 ensures that there is a maximal solution of $(\underline{16})$ in $C\left(\left[0, T_{\max }\right), W^{1, q}(\Omega)\right) \cap C\left(\left(0, T_{\max }\right), W^{2, q}(\Omega)\right)$ for $q>2$.

We begin the proof by the following lemmas which gives some estimates on $u$ and $\boldsymbol{\omega}$. 
Lemma 4.1. Let the same assumptions as that of Theorem 2.2 hold, and $u$ be the nonnegative maximal solution of (16). Then for all $T>0$ there exists $C_{1}(T)>0$, such that $u$ and $\boldsymbol{\omega}$ satisfy the following estimates

$$
\|u(t)\|_{2}^{2}+\int_{0}^{t}\|\nabla u(s)\|_{2}^{2} d s \leq C_{1}(T), \text { for all } t \in[0, T] \cap\left[0, T_{\max }\right),
$$

and

$$
\int_{0}^{t}\left(\|\nabla \cdot \boldsymbol{\omega}(s)\|_{2}^{2}+\|\boldsymbol{\omega}(s)\|_{2}^{2}\right) d s \leq C_{1}(T) \text { for all } t \in[0, T] \cap\left[0, T_{\max }\right) .
$$

Proof. We multiply the first equation in (16) by $2 u$ and integrate it over $\Omega$, to obtain

$$
\frac{\mathrm{d}}{\mathrm{d} t} \int_{\Omega}|u|^{2} d x=-2 \delta \int_{\Omega}|\nabla u|^{2} d x+2 \int_{\Omega} u \boldsymbol{\omega} \cdot \nabla u d x+2 r \int_{\Omega} u^{2} E(u) d x .
$$

We multiply now the second equation in (16) by $\boldsymbol{\omega}$ and integrate it over $\Omega$. We note that the boundary conditions for $\boldsymbol{\omega}$ guarantee that $\boldsymbol{\omega}$ is tangent to $\partial \Omega$ while $\partial_{\boldsymbol{n}} \boldsymbol{\omega}$ is normal to $\partial \Omega$. Consequently, $\partial_{\boldsymbol{n}} \boldsymbol{\omega} \cdot \boldsymbol{\omega}=0$ on $\partial \Omega$ and it follows from an integration by parts that

$$
\begin{aligned}
-\varepsilon \int_{\Omega} \Delta \boldsymbol{\omega} \cdot \boldsymbol{\omega} d x & =\varepsilon \int_{\Omega}|\nabla \cdot \boldsymbol{\omega}|^{2} d x-\varepsilon \int_{\partial \Omega}\left[\left(\nabla \omega_{1} \cdot \boldsymbol{n}\right) \omega_{1}+\left(\nabla \omega_{2} \cdot \boldsymbol{n}\right) \omega_{2}\right] d \sigma \\
& =\varepsilon \int_{\Omega}|\nabla \cdot \boldsymbol{\omega}|^{2} d x .
\end{aligned}
$$

We thus obtain

$$
\varepsilon \int_{\Omega}|\nabla \cdot \boldsymbol{\omega}|^{2} d x+\int_{\Omega}|\boldsymbol{\omega}|^{2} d x=-2 \int_{\Omega} u \boldsymbol{\omega} \cdot \nabla u d x+(a+1) \int_{\Omega} \boldsymbol{\omega} \cdot \nabla u d x .
$$

At this point we notice that the cubic terms on the right hand side of (19) and (20) cancel one with the other, and summing (20) and (19) we obtain

$$
\frac{\mathrm{d}}{\mathrm{d} t}\|u\|_{2}^{2}+\varepsilon\|\nabla \cdot \boldsymbol{\omega}\|_{2}^{2}+\|\boldsymbol{\omega}\|_{2}^{2}+2 \delta\|\nabla u\|_{2}^{2}=2 r \int_{\Omega} u^{2} E(u) d x+(a+1) \int_{\Omega} \boldsymbol{\omega} \cdot \nabla u d x .
$$

We integrate by parts and use Cauchy-Schwarz inequality to obtain

$$
(a+1) \int_{\Omega} \boldsymbol{\omega} \cdot \nabla u d x=-(a+1) \int_{\Omega} u \nabla \cdot \boldsymbol{\omega} d x \leq \frac{(a+1)^{2}}{2 \varepsilon}\|u\|_{2}^{2}+\frac{\varepsilon}{2}\|\nabla \cdot \boldsymbol{\omega}\|_{2}^{2} .
$$

On the other hand, $u^{2} E(u) \leq 0$ if $u \notin(a, 1)$ so that

$$
\int_{\Omega} u^{2} E(u) d x \leq|\Omega|(1-a) .
$$

The previous inequalities give

$$
\frac{\mathrm{d}}{\mathrm{d} t}\|u\|_{2}^{2}+\frac{\varepsilon}{2}\|\nabla \cdot \boldsymbol{\omega}\|_{2}^{2}+\|\boldsymbol{\omega}\|_{2}^{2}+2 \delta\|\nabla u\|_{2}^{2} \leq \frac{(a+1)^{2}}{2 \varepsilon}\|u\|_{2}^{2}+2|\Omega| r(1-a) .
$$

Therefore, for all $T>0$ there exists $C_{1}(T)$ such that (17) and (18) hold.

Lemma 4.2. Let the same assumptions as that of Theorem 2.2 hold, and $u$ be the nonnegative maximal solution of (16). Then for all $T>0$ there exists $C_{2}(T, p)>0$, such that for $p \geq 2$

$$
\begin{gathered}
\|u(t)\|_{p} \leq C_{2}(T, p) \text { for all } t \in[0, T] \cap\left[0, T_{\max }\right) \\
\int_{0}^{t}\left\|\nabla u^{\frac{p}{2}}(s)\right\|_{2}^{2} d s \leq C_{2}(T, p) \text { for all } t \in[0, T] \cap\left[0, T_{\max }\right) .
\end{gathered}
$$


Proof. We multiply the first equation in (16) by $p u^{p-1}$, integrate with respect to $x$, and integrate by parts. The boundary terms vanish and we obtain

$$
\begin{aligned}
\frac{\mathrm{d}}{\mathrm{d} t}\|u\|_{p}^{p} & \leq \frac{-4 \delta(p-1)}{p}\left\|\nabla u^{\frac{p}{2}}\right\|_{2}^{2}-(p-1) \int_{\Omega} \nabla \cdot \boldsymbol{\omega} u^{p} d x \\
& +r p \int_{\Omega} u^{p-1} E(u) d x .
\end{aligned}
$$

By Cauchy-Schwarz inequality we obtain

$$
\begin{aligned}
\frac{\mathrm{d}}{\mathrm{d} t}\|u\|_{p}^{p} & \leq \frac{-4 \delta(p-1)}{p}\left\|\nabla u^{\frac{p}{2}}\right\|_{2}^{2}+(p-1)\|\nabla \cdot \boldsymbol{\omega}\|_{2}\left\|u^{\frac{p}{2}}\right\|_{4}^{2} \\
& +\operatorname{rp} p(1-a)|\Omega| .
\end{aligned}
$$

Using the Gagliardo-Nirenberg inequality (13) we have

$$
\left\|u^{\frac{p}{2}}\right\|_{4} \leq C\left\|u^{\frac{p}{2}}\right\|_{W^{1,2}}^{\frac{1}{2}}\left\|u^{\frac{p}{2}}\right\|_{2}^{\frac{1}{2}} .
$$

Substituting (24) in (23), and by Young inequality we obtain

$$
\begin{aligned}
\frac{\mathrm{d}}{\mathrm{d} t}\|u\|_{p}^{p} & \leq \frac{-4 \delta(p-1)}{p}\left\|\nabla u^{\frac{p}{2}}\right\|_{2}^{2}+C(p-1)\|\nabla \cdot \boldsymbol{\omega}\|_{2}\left\|u^{\frac{p}{2}}\right\|_{W^{1,2}}\left\|u^{\frac{p}{2}}\right\|_{2} \\
& +r p(1-a)|\Omega| \\
& \leq \frac{-4 \delta(p-1)}{p}\left\|\nabla u^{\frac{p}{2}}\right\|_{2}^{2}+\frac{2 \delta(p-1)}{p}\left\|u^{\frac{p}{2}}\right\|_{W^{1,2}}^{2} \\
& +C(p)\|\nabla \cdot \boldsymbol{\omega}\|_{2}^{2}\left\|u^{\frac{p}{2}}\right\|_{2}^{2}+C(p) \\
& \leq \frac{-2 \delta(p-1)}{p}\left\|\nabla u^{\frac{p}{2}}\right\|_{2}^{2}+C(p)\|u\|_{p}^{p}+C(p)\|\nabla \cdot \boldsymbol{\omega}\|_{2}^{2}\|u\|_{p}^{p}+C(p) .
\end{aligned}
$$

Next, integrating the above inequality in time, and using (18) yield that there exists $C_{2}(T, p)$ such that $(21)$ and (22) hold.

Lemma 4.3. Let the same assumptions as that of Theorem 2.2 hold, and $u$ be the nonnegative maximal solution of (16). Then for all $T>0$ there exists $C_{3}(T)>0$, such that

$$
\|\nabla u(t)\|_{2} \leq C_{3}(T) \text { for all } t \in[0, T] \cap\left[0, T_{\max }\right) .
$$

Proof. We multiply the first equation in (16) by $-\Delta u$, integrate over $\Omega$, and use CauchySchwarz, and Young inequalities and (21) to obtain

$$
\begin{aligned}
\frac{1}{2} \frac{\mathrm{d}}{\mathrm{d} t}\|\nabla u\|_{2}^{2} & =-\delta\|\Delta u\|_{2}^{2}+\int_{\Omega}(\nabla u \cdot \boldsymbol{\omega}+\nabla \cdot \boldsymbol{\omega} u) \Delta u d x-r \int_{\Omega} u(1-u)(u-a) \Delta u d x \\
& \leq-\delta\|\Delta u\|_{2}^{2}+\frac{\delta}{2}\|\Delta u\|_{2}^{2}+C \int_{\Omega}|\nabla u|^{2}|\boldsymbol{\omega}|^{2} d x \\
& +C \int_{\Omega}|\nabla \cdot \boldsymbol{\omega}|^{2}|u|^{2} d x+C r\|u(1-u)(u-a)\|_{2}^{2}+\frac{\delta}{4}\|\Delta u\|_{2}^{2} \\
& \leq-\frac{\delta}{4}\|\Delta u\|_{2}^{2}+C \int_{\Omega}|\nabla u|^{2}|\boldsymbol{\omega}|^{2} d x+C \int_{\Omega}|\nabla \cdot \boldsymbol{\omega}|^{2}|u|^{2} d x+C(T) .
\end{aligned}
$$

To go further requires to improve the estimate on $\boldsymbol{\omega}$ and $\nabla \cdot \boldsymbol{\omega}$. For that purpose, we use Lemma $\underline{4.1}$ and Lemma $\underline{4.2}$ for $p=4$ to obtain for all $T>0$ 


$$
\int_{0}^{t}\|\nabla E(u)(s)\|_{2}^{2} d s \leq(a+1)^{2} \int_{0}^{t}\|\nabla u(s)\|_{2}^{2} d s+4 \int_{0}^{t}\|u \nabla u(s)\|_{2}^{2} d s \leq C_{2}(T),
$$

for all $t \in[0, T] \cap\left[0, T_{\max }\right)$. Consequently, $\nabla E(u)$ is bounded in $L^{2}((0, t) \times \Omega)$. By Theorem 3.1 and the continuous embedding of $W^{2,2}(\Omega)$ in $W^{1,4}(\Omega)$, and $W^{1,4}(\Omega)$ in $L^{\infty}(\Omega)$, we have

$$
\|\boldsymbol{\omega}\|_{\infty}+\|\nabla \cdot \boldsymbol{\omega}\|_{4} \leq C\|\boldsymbol{\omega}\|_{W^{1,4}} \leq C\|\boldsymbol{\omega}(s)\|_{W^{2,2}} \leq C\|\nabla E(u)\|_{2},
$$

which together with (27) implies that

$$
\int_{0}^{t}\left(\|\boldsymbol{\omega}(s)\|_{\infty}^{2}+\|\nabla \cdot \boldsymbol{\omega}(s)\|_{4}^{2}\right) d s \leq C \int_{0}^{t}\|\nabla E(u)(s)\|_{2}^{2} d s \leq C_{2}(T),
$$

for all $t \in[0, T] \cap\left[0, T_{\max }\right)$. Using Hölder Inequality , (26) becomes

$$
\frac{1}{2} \frac{\mathrm{d}}{\mathrm{d} t}\|\nabla u\|_{2}^{2} \leq-\frac{\delta}{4}\|\Delta u\|_{2}^{2}+C\|\boldsymbol{\omega}\|_{\infty}^{2} \int_{\Omega}|\nabla u|^{2} d x+C\|\nabla \cdot \boldsymbol{\omega}\|_{4}^{2}\|u\|_{4}^{2}+C(T) .
$$

Next we integrate the above inequality in time, and use (21) for $p=4$, and (28) to obtain

$$
\|\nabla u(t)\|_{2}^{2} \leq\left\|\nabla u_{0}\right\|_{2}^{2}+C \int_{0}^{t}\|\boldsymbol{\omega}(s)\|_{\infty}^{2}\|\nabla u(s)\|_{2}^{2} d s+C(T)
$$

using (28) again, we have thus proved (25).

Lemma 4.4. Let the same assumptions as that of Theorem 2.2 hold, and $u$ be the nonnegative maximal solution of (16). Then for all $T>0$ there exists $C_{4}(T)>0$, such that

$$
\|\boldsymbol{\omega}(t)\|_{\infty} \leq C_{4}(T), \text { for all } t \in[0, T] \cap\left[0, T_{\max }\right) .
$$

Proof. It follows from (21), (25) and Hölder inequality, that there exists $C(T)>0$ such that

$$
\|(-2 u+a+1) \nabla u\|_{\frac{3}{2}} \leq C(T)\|-2 u+a+1\|_{6}\|\nabla u\|_{2} \leq C(T) .
$$

Consequently, Theorem $\underline{3.1}$ ensures that

$$
\|\boldsymbol{\omega}\|_{W^{2, \frac{3}{2}}} \leq C\|[-2 u+a+1] \nabla u\|_{\frac{3}{2}} \leq C(T) \text {, for all } t \in[0, T] \cap\left[0, T_{\max }\right) .
$$

Using the continuous embedding of $W^{2, \frac{3}{2}}(\Omega)$ in $L^{\infty}(\Omega)$ we have thus proved (29).

Next, thanks to lemma A.1 in [8] we can derive a uniform bound for $u$.

Lemma 4.5. Let the same assumptions as that of Theorem $\underline{2.2}$ hold, and $u$ be the nonnegative maximal solution of (16). Then for all $T>0$ there exists $C_{5}(T)>0$, such that

$$
\|u(t)\|_{\infty} \leq C_{5}(T), \text { for all } t \in[0, T] \cap\left[0, T_{\max }\right) .
$$

Proof. We can see that the function $u$ solves

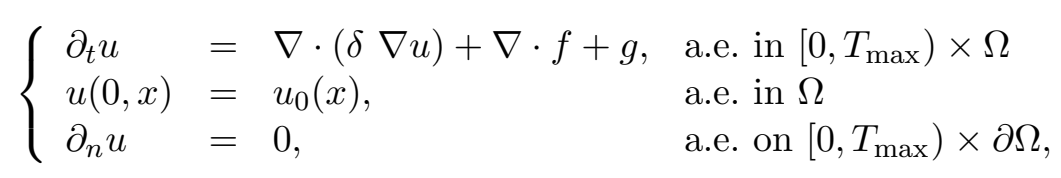


where $f=-u \boldsymbol{\omega}$ and $g=r u E(u)$.

By the regularity $(\underline{10})$ of $u$, and by the continuous embeddings of $W^{1, p}(\Omega)$ in $C(\bar{\Omega})$ and of $W^{2, p}(\Omega)$ in $C(\bar{\Omega})$ for $p>2$ we obtain that $f=-u \boldsymbol{\omega} \in C\left(\left(0, T_{\max }\right) ; C(\bar{\Omega})\right)$ and $\nabla f \in C\left(\left(0, T_{\max }\right) ; C(\bar{\Omega})\right)$. Using $(\underline{10})$ and the continuous embeddings of $W^{1, p}(\Omega)$ in $C(\bar{\Omega})$ for $p>2$ again, we see that $g=r u E(u) \in C\left(\left(0, T_{\max }\right) \times \bar{\Omega}\right)$. On the other hand, $f \cdot n=-u \boldsymbol{\omega} \cdot n=0$ on $\partial \Omega \times\left(0, T_{\max }\right)$.

Thanks to (21), we have

$$
\|u(t)\|_{p_{0}} \leq C(T), \text { for all } t \in[0, T] \cap\left[0, T_{\max }\right)
$$

where $p_{0}=9$, while $(21)$ and $(29)$ yield

$$
\|f(t)\|_{q_{1}} \leq C(T) \text { and }\|g\|_{q_{2}} \leq C(T) \text {, for all } t \in[0, T] \cap\left[0, T_{\max }\right)
$$

where $q_{1}>4$ and $q_{2}=3>2$. Since $p_{0}>1-\frac{3 q_{1}-4}{q_{1}-3}$ and $p_{0}=9>0$, we can apply lemma A.1 in [8], and the estimate (30) holds.

Lemma 4.6. Let the same assumptions as that of Theorem 2.2 hold, and $u$ be the nonnegative maximal solution of (16). Then for all $T>0$ there exists $C_{6}(T)>0$, such that

$$
\|\nabla u(t)\|_{q} \leq C_{6}(T), \text { for all } t \in[0, T] \cap\left[0, T_{\max }\right) .
$$

Proof. Using (11),(12), (30) and (29) we have for $q>2$

$$
\begin{aligned}
\|\nabla u(t)\|_{q} & \leq C\left\|u_{0}\right\|_{W^{1, q}}+C_{1} \int_{0}^{t}(t-s)^{-\frac{1}{2}}\|\nabla \cdot(u \boldsymbol{\omega})(s)\|_{q} d s \\
& +r C_{1} \int_{0}^{t}\|\nabla(u E(u))(s)\|_{q} d s \\
& \leq C\left\|u_{0}\right\|_{W^{1, q}}+C_{1} \int_{0}^{t}(t-s)^{-\frac{1}{2}}\|u(s)\|_{\infty}\|\nabla \cdot \boldsymbol{\omega}(s)\|_{q} d s \\
& +C_{1} \int_{0}^{t}(t-s)^{-\frac{1}{2}}\|\boldsymbol{\omega}(s)\|_{\infty}\|\nabla u(s)\|_{q} d s \\
& +r C_{1} \int_{0}^{t}\left\|\left(-3 u^{2}+2(a+1) u-a\right)(s)\right\|_{\infty}\|\nabla u(s)\|_{q} d s . \\
& \leq C(T)\left(1+\int_{0}^{t}(t-s)^{-\frac{1}{2}}\left(\|\nabla \cdot \boldsymbol{\omega}(s)\|_{q}+\|\nabla u(s)\|_{q}\right) d s+\int_{0}^{t}\|\nabla u(s)\|_{q} d s\right) .
\end{aligned}
$$

By Theorem 3.1 we have

$$
\|\nabla \cdot \boldsymbol{\omega}(s)\|_{q} \leq\|\boldsymbol{\omega}(s)\|_{W^{2, q}} \leq K(q)\|(-2 u+a+1)(s)\|_{\infty}\|\nabla u(s)\|_{q} .
$$

Substituting (34) into (33) and using (30) we obtain

$$
\|\nabla u(t)\|_{q} \leq C(T)\left(1+\int_{0}^{t}(t-s)^{-\frac{1}{2}}\|\nabla u(s)\|_{q} d s+\int_{0}^{t}\|\nabla u(s)\|_{q} d s\right) .
$$

Now, we obtain (32) by applying Lemma $\underline{3.3}$ with $\beta=0$, and $\alpha=\frac{1}{2}$.

Thanks to these lemmas we can now prove the main Theorem 2.2 
Proof of Theorem 2.2. For all $T>0$, Lemma 4.2 and Lemma $\underline{4.5}$ ensure that for $q>2$

$$
\|u(t)\|_{W^{1, q}} \leq C(T), \text { for all } t \in[0, T] \cap\left[0, T_{\max }\right)
$$

which guarantees that $u$ cannot explode in $W^{1, q}(\Omega)$ in finite time and thus that $T_{\max }=\infty$.

\subsection{Monostable case}

For this choice of $E$, system (1)-( $\underline{3})$ now reads

$$
\left\{\begin{array}{lll}
\partial_{t} u & =\delta \Delta u-\nabla \cdot(u \boldsymbol{\omega})+r u(1-u), & x \in \Omega, t>0 \\
-\varepsilon \Delta \boldsymbol{\omega}+\boldsymbol{\omega} & =-\nabla u, & x \in \Omega, t>0 \\
\partial_{\boldsymbol{n}} u=0 & , \quad \boldsymbol{\omega} \cdot \boldsymbol{n}=\partial_{\boldsymbol{n}} \boldsymbol{\omega} \times \boldsymbol{n}=0, & x \in \partial \Omega, t>0 \\
u(0, x) & =u_{0}(x), & x \in \Omega,
\end{array}\right.
$$

when $u_{0} \in W^{1, q}(\Omega)$ for some $q>2$. Since $E \in C^{2}(\mathbb{R})$, Theorem $\underline{3.2}$ ensures that there is a maximal solution of $(\underline{35})$ in $C\left(\left[0, T_{\max }\right), W^{1, q}(\Omega)\right) \cap C\left(\left(0, T_{\max }\right), W^{2, q}(\Omega)\right)$ for $q>2$.

To prove Theorem 2.3 we need to prove the following lemma

Lemma 4.7. Let the same assumptions as that of Theorem 2.3 hold, and let $u$ be the maximal solution of (35). Then for all $T>0$, there exists $C_{7}(T)>0$ such that

$$
\int_{0}^{t}\left(\|\boldsymbol{\omega}(s)\|_{2}^{2}+\|\nabla \cdot \boldsymbol{\omega}(s)\|_{2}^{2}\right) d s \leq C_{7}(T), \text { for all } t \in[0, T] \cap\left[0, T_{\max }\right) .
$$

Proof. The proof goes as follows. On the one hand, we multiply the first equation in (35) by $(\log u+1)$ and integrate it over $\Omega$, the boundary terms vanish. Since $u(1-u) \log u \leq 0$ and $u(1-u) \leq 1$,

$$
\begin{aligned}
\frac{\mathrm{d}}{\mathrm{d} t} \int_{\Omega} u \log u d x & =-\int_{\Omega}(\delta \nabla u-u \boldsymbol{\omega}) \cdot\left(\frac{1}{u} \nabla u\right) d x+r \int_{\Omega} u(1-u)(\log u+1) d x \\
& \leq-\int_{\Omega} \frac{\delta}{u}|\nabla u|^{2} d x+\int_{\Omega} \boldsymbol{\omega} \cdot \nabla u d x+|\Omega| r
\end{aligned}
$$

On the other hand, we multiply the second equation in (35) by $\omega$, integrate it over $\Omega$. We note that the boundary conditions for $\boldsymbol{\omega}$ guarantee that $\boldsymbol{\omega}$ is tangent to $\partial \Omega$ while $\partial_{\boldsymbol{n}} \boldsymbol{\omega}$ is normal to $\partial \Omega$. Consequently, $\partial_{\boldsymbol{n}} \boldsymbol{\omega} \cdot \boldsymbol{\omega}=0$ on $\partial \Omega$ and it follows from an integration by parts that

$$
\varepsilon \int_{\Omega}|\nabla \cdot \boldsymbol{\omega}|^{2} d x+\int_{\Omega}|\boldsymbol{\omega}|^{2} d x=-\int_{\Omega} \boldsymbol{\omega} \cdot \nabla u d x
$$

Adding (38) and (37) yields

$$
\frac{\mathrm{d}}{\mathrm{d} t} \int_{\Omega} u \log u d x+\varepsilon\|\nabla \cdot \boldsymbol{\omega}\|_{2}^{2}+\|\boldsymbol{\omega}\|_{2}^{2} \leq-4 \delta \int_{\Omega}|\nabla \sqrt{u}|^{2} d x+|\Omega| r .
$$

Finally, (36) is obtained by a time integration of (39).

Proof of Theorem 2.3. Thanks to ( $\underline{36})$, we now argue as in the proof of Lemma $\underline{4}$.2, Lemma 4.3. Lemma $\underline{4.4}$, Lemma $\underline{4.5}$ and Lemma $\underline{4.6}$ to get that for $q>2$

$$
\|u(t)\|_{\infty}+\|\nabla u(t)\|_{q} \leq C(T), \text { for all } t \in[0, T] \cap\left[0, T_{\max }\right) .
$$

Thus, the maximal solution $u$ of $(\underline{35})$ cannot explode in finite time. 


\section{Acknowledgment}

I thank Philippe Laurençot for fruitful discussion.

\section{References}

[1] H. Amann. Linear and quasilinear parabolic problems . Volume I . Abstract linear theory, (1995)

[2] T. Cazenave. A. Haraux. An Introduction to semilinear evolution equations. Oxford lecture series in mathematics and its applications, (2006).

[3] J. P. Dias. A simplified variational model for the bidimensional coupled evolution equations of a nematic liquid crystal. J. Math. Anal. Appl. 67 (1979), no. 2, 525-541.

[4] J. P. Dias. Un problème aux limites pour un système d'équations non linéaires tridimensionnel. Bolletino, U. M.I. (5) 16-B (1979), 22-31.

[5] P. Grindrod. Models of individual aggregation or clustering in single and multi-species communities. J. Math. Biol. (26) (1988) 651-660.

[6] E. Nasreddine. Well-posedness for a model of individual clustering. ArXiv:1211.2969v1 [math.AP] (2012).

[7] M. Schoenauer. Quelques résultats de régularité pour un système elliptique avec conditions aux limites couplées. Annales de la Faculté des Sciences de Toulouse 5e série, tome 2, n2(1980), 125-135.

[8] Y. Tao, M. Winkler. Boundedness in a quasilinear parabolic-parabolic Keller-Segel system with subcritical sensitivity. J. Differential Equations 252 (2012), no. 1, 692715. 